\title{
Honey Sterilization and Mead Production
}

\author{
Andreea C. ŞARBA*1), Liviu Al. MĂRGHITAŞ⿻ి', Adrian TIMAR²) \\ ${ }^{1)}$ Faculty of Natural and Agricultural Sciences, University of Agricultural Sciences and Veterinary \\ Medicine, 3-5 Mănăştur Street, 400372 Cluj-Napoca, Romania \\ ${ }^{2)}$ Faculty of Environmental Protection, University of Oradea, 26 Gen. Magheru Bd., Romania \\ *Corresponding author, email: andreea.sarba@yahoo.com
}

Bulletin UASVM Animal Science and Biotechnologies 71(2) / 2014,

Print ISSN 1843-5262; Electronic ISSN 1843-536X

DOI:10.15835/buasvmcn-asb:10514

\begin{abstract}
The objective of this study was to evaluate the effects of fast freezing on honey, the main raw material of mead making. Polyfloral honey was subjected to fast freezing process and after that fermented into honey wine. The fast freezing process was made at a temperature of $-80^{\circ}$ for one hour. Microbiological condition of honey was tested in order to prevent the contamination of honey must used in fermentation. The sample was inoculated on DRBC agar cultures and compared with a non-freezed sample of polyfloral honey. On freezed agar plate there were not identified any susceptible colonies of yeasts and mold. No significant difference in organoleptic properties due to freezing treatments was observed in the final product -mead (honey wine).
\end{abstract}

Keywords: mead, honey, sterilization, fast freeze, fermentation, honey wine

Introduction. Honey, the sweet substance produced by honey bees, has been used for centuries to prepare traditional, homemade drinks. Honey can be fermented to produce different types of mead (honey wine), sherry type wine, sparkling wine and fruit-honey wine, which may have different flavors depending the floral source of the honey and additives and yeast used in fermentation (Gupta and Sharma, 2009).

Mead contains 8-18\% (v/v) ethanol, obtained by the alcoholic fermentation of diluted bee honey with an appropriate amount of water. Mead fermentation is a time-consuming process, often taking several months, and the fermentation rate depends on several factors, especially on honey variety, yeast strain, yeast nutrition, control of $\mathrm{pH}$ (Navrátil et al., 2001).

Honey is the main component of mead making process and the success of fermentation process is crucial in mead production. During the fermentation scheme, we have two main control points noted as critical process points. The first is during the fermentation process and it is due to possible microbiological contamination of the main raw material, which is honey. The basic method of preparing and sterilize the raw material is by boiling it. The advantage of boiling the must is that proteins and impurities are more easily coagulated and removed. But this method comes with notable disadvantages such as that volatile aromatic compounds are driven off and the final product doesn't gather organoleptic properties, which were targeted. The no-boil approach that we think off was rapid freezing honey technique, which has been shown to be an attractive method for various raw materials. Fast freeze was developed to overcome the loss of compounds responsible for flavor and aroma, which are lost during conventional sterilization process of boiling. The main characteristic of freezing is virtual absence of air during processing. The absence of air and low temperature prevent deterioration due to oxidation or chemical modification of the honey.

Aims and objectives. The ideal situation for mead making is to have only one species of microorganism ever present in the wine, which is the selected yeast for fermentation. However, by the very nature of the sheer abundance of 
microorganism present in the environment this ideal situation is never realized (Spedding, 2000). The aim of this study was to sterilize the raw material used in wine production, which is honey in order to prevent possible faults due to microbiological contamination during fermentation process.

Materials and methods. This study was conducted in the Department of Technological Sciences-Animal Breeding, of UASVM in partnership with Food-biochemistry Laboratory of the Faculty of Environmental Protection of the University of Oradea in June 2014. We used a type of polyfloral honey obtained from a traditional beekeeper of Bihor County in May 2014. For this experiment, we had prepared 2 samples of honey. We had fast freezed one sample of honey in Sanyo Cryogenic chest freezer MDF 192 at a temperature of $-80^{\circ} \mathrm{C}$ for an hour. The two samples of honey, the raw and the freeze honey were diluted at $10^{-1}$ and inoculated on DRBC agar cultures. All samples were performed in duplicate.

Results and discussion. It is well known that by controlling environment nutrients like, oxygen, water, temperature, acidity and $\mathrm{pH}$, we can operate the growth of microorganism by altering their growth rate or eliminate them from the environment completely. In our case, we've decided to act over the temperature. The effect of low temperatures on microorganisms depends on the particular microbe and the intensity of application. For example, at temperatures of ordinary refrigerators like $0^{\circ} \mathrm{C}$, the metabolic rate of some microbes is so reduced that they cannot reproduce or synthesize toxins. In other words, ordinary refrigeration has bacteriostatic effect, but does not kill many microorganisms, ordinary freezing do not achieve sterilization. That's why we've considered fast freezing our raw material. It is known that microorganisms, especially yeasts and mold don't survive at a wide temperature range, especially very low temperatures such as $-80^{\circ} \mathrm{C}$.

Considering all the information above and the experiment we've conducted the results were very concluding: on freezed agar plate there were not identified any susceptible colonies of yeasts and mold. Also, original organoleptic and chemical were not affected by rapid freezing process.

Conclusion. The raw material used in mead production -honey- can be infected with many bacterial species and wild yeast that can alter organoleptic or physical and chemical properties of the final product. Every brewer must therefore be aware of the types of organisms, the kinds of spoilage they can render on the final product, and how to effectively remove them as potential contaminants (Spedding, 2000). So far, in our study, this procedure is the most viable for sterilize raw material, honey, due to obtain and maximize the productivity of mead.

Acknowledgements. This paper was published under the frame of European Social Found, Human Resources Development Operational Programme 2007-2013, project no. POSDRU/159/1.5/S/132765

\section{REFERENCES}

1. Gupta JK and Sharma R (2009). Production technology and quality characteristics of mead and fruit-honey wines: A review. Natural product Radiance, 8(4), 345-355.

2. Navrátil M, Sturdik E, Gemeiner P (2001). Batch and continuous mead production with pectate imobilised, ethanol-tolerant yeasts. Biotechnology Letters 23, 977982.

3. Spedding (2000). Microbiological media for bacteria and wild yeast detection in the brewery - seminar. 